\title{
Generation of an Effective Training Feature Vector using VQ for Classification of Image Database
}

\author{
H. B. Kekre \\ Professor, Computer \\ Engineering, Mukesh Patel \\ School of Technology \\ Management and Engineering, \\ NMIMS University, Vileparle(w) \\ Mumbai 400056, India
}

\author{
Tanuja K. Sarode \\ Associate Professor, Computer \\ Engineering, Thadomal Shahani \\ Engineering College, \\ Bandra(W), Mumbai 400050, \\ India
}

\author{
Jagruti K. Save \\ Ph.D. Research Scholar, \\ MPSTME, NMIMS University, \\ Associate Professor, Computer \\ Engineering, Fr. C. Rodrigues \\ College of Engineering, \\ Bandra(W), Mumbai 400050, \\ India
}

\begin{abstract}
In supervised classification of image database, feature vectors of images with known classes, are used for training purpose. Feature vectors are extracted in such a way that it will represent maximum information in minimum elements. Accuracy of classification highly depends on the content of training feature vectors and number of training feature vectors. If the number of training images increases then the performance of classification also improves. But it also leads to more storage space and computation time. The main aim of this research is to reduce the number of feature vectors in an effective way so as to reduce memory space required and computation time as well as to increase an accuracy. This paper proposes three major steps for automatic classification of image database. First step is the generation of feature vector of an image using column transform, row mean vector and fusion method. Then vector Quantization (code book size 4,8 and 16) is applied to reduce the number of training feature vectors per class and generate an effective and compact representation of them. Finally nearest neighbor classification algorithm is used as a classifier. The experiments are conducted on augmented Wang database. The results for various transforms, different similarity measures, varying sizes of feature vector, three code book sizes and different number of training images, are analyzed and compared. Results show that the proposed method increases accuracy in most of the cases.
\end{abstract}

\section{General Terms}

Image Classification, Vector quantization, Algorithms, Image Database.

\section{Keywords}

Supervised Classification, Row Mean Vector, Similarity Measures, Nearest Neighbor Classifier, Feature Vector.

\section{INTRODUCTION}

Automatic Image Classification has become more important with the development of Internet and the growth in the size of image databases. It is a process of assigning images to a number of predefined categories. For large databases, classification or categorization of images is an useful preprocessing step for image retrieval system. A successful classification of images will greatly enhance the performance of content-based image retrieval systems by filtering out images from irrelevant classes during matching (A.Vailaya et al. 1998)[1]. Due to the content heterogeneity that exist among the different images which are downloaded from the internet, their automatic classification has become a great challenge. Classification of images is of two types : Supervised and Unsupervised. In Supervised classification, the training set (set of images with their classes) is available. In unsupervised classification, no such training set is available. Unsupervised classification is also known as clustering. This paper proposes the technique for supervised classification. Most of the classification systems generate image feature vectors for dimension reduction and as a representation of an image. Feature extraction is an important issue for generic image classification. So lot of research has been done in this area. N.Manshor et al. 2012, proposed the combination of different low level features with local features for improving the performance of object class recognition[2]. H.Nakayama et al. 2009, increased the performance of global features by using local feature correlation[3]. D.Choudhary et al. 2013, has shown that the wavelet features can be used to generate feature vector[4]. The combination of local and global properties can also be considered to generate feature vectors (W.H.Cho et al. 2013)[5]. These extracted features are applied to classifiers such as nearest neighbor classifier(O. Boiman et al. 2008)[6], decision tree classifier and Bayes classifier. Artificial neural network (ANN) and Support vector machine (SVM) are also popular methods for classification (M.W.Ashour et al. 2013)[7]. Li Fei-Fei et al. 2005, proposed a Bayesian hierarchical model to learn and recognize natural scene categories[8]. S.D.M. Raja et al. 2011, has given comparison of ANN and SVM based classifiers[9].

In this paper we proposed a system in which initially a transform is applied to the columns of an image. Image transforms are mathematical operations which convert images from one representation to another. The Fourier Transform is an important image processing tool which is used to decompose an image into its sine and cosine components (O.Brigham et al. 1967)[10]. The main idea of the Fourier transform is that a complex signal can be expressed as the sum of sines and cosines of different amplitudes. Most unitary transforms pack a large fraction of the energy of the image into relatively few of the transform coefficients. This property makes it useful for compression and dimension reduction. We have used various transforms such as discrete Fourier transform (DFT), discrete cosine transform (DCT) (N.Ahmed et al. (1974)[11], discrete sine transform (DST) (A.K.Jain et al. 1976, H.B.Kekre et al. 1978)[12][13], discrete Hartley transform (DHT) (Hartley,R.V.L. 1942)[14], discrete Walsh transform (DWT) (J.L.Walsh 1923)[15] and discrete Kekre Transform (DKT) (H.B.Kekre et al. 2009)[16]. The process of calculating mean of every row of transformed image gives row mean vectors of an image over R,G,B planes. Using fusion of these vectors, feature vector is generated. To get high accuracy for large database, the number of training images should be sufficiently large. But it also causes more storage space and computation time. To deal with this 
problem, we have suggested to generate an effective representation from those training images. This representation contains few number of training feature vectors. For classification stage, nearest neighbor classifier is implemented. To find the closest feature vector, different similarity measures such as Euclidean distance, Manhattan distance(E.Deza et al 2006)[17] (John P. et al. 1995)[18], Cosine correlation similarity and Bray-Curtis similarity (S.Santini et al. 1999)[19], (H.B.kekre et al. 2012)[20] have been used. The paper is organized as follows : Section 2 explains LBG (Linde-Buzo-Gray,1980)[21] (R.M.Gray, 1984)[22] vector quantization method. Section 3 gives detailed procedure of proposed system. Section 4 shows all the results of implementation followed by conclusions and future scope in section 5 and references in section 6 .

\section{LINDE-BUZO-GRAY ALGORITHM}

In 1980, Linde et al. proposed a Generalized Lloyd Algorithm (GLA) which is also called Linde-Buzo-Gray (LBG) algorithm. This algorithm is applied on all training feature vectors of each class. Consider the classes are numbered as $\mathrm{C}_{1}, \mathrm{C}_{2}, \ldots, \mathrm{C}_{20}$.

The LBG algorithm steps are as follows :

For Class $\mathrm{C}_{1}$ to $\mathrm{C}_{20}$ do

\section{BEGIN}

1. All training feature vectors of the class belong to one cluster.

2. Calculate the centroid of all vectors. This is the first code vector.

3. Addition and subtraction of constant error with the code vector forms two trial code vectors say $\mathrm{v}_{1}$ and $\mathrm{v}_{2}$. Assume these vectors represent two clusters.

4. Find the closeness for each training vector in a cluster with these two trial code vectors. Put the feature vectors in an appropriate cluster. Thus initial single cluster is divided into two clusters.

5. Calculate the centroid for each cluster. These are two code vectors.

6. Repeat the steps 3 to step 5 for each cluster till the desired number of code vectors generated. After every iteration each cluster gets divided into two clusters.

7. Store these code vectors.

\section{END}

Figure 1(a) \& (b) shows the LBG procedure representation for two dimensional training vectors.

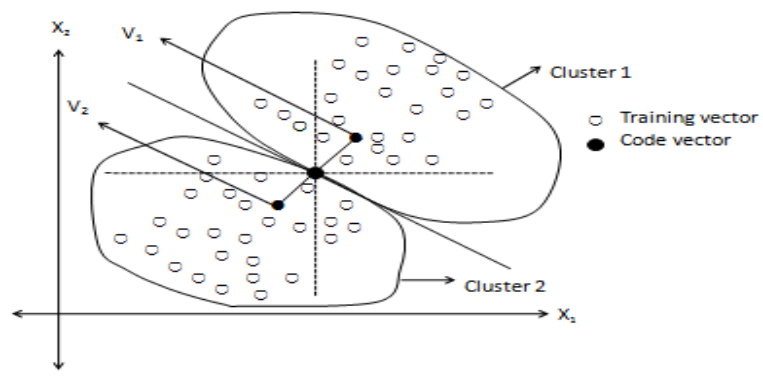

Figure 1(a) LBG for two dimensional case after two clusters formation

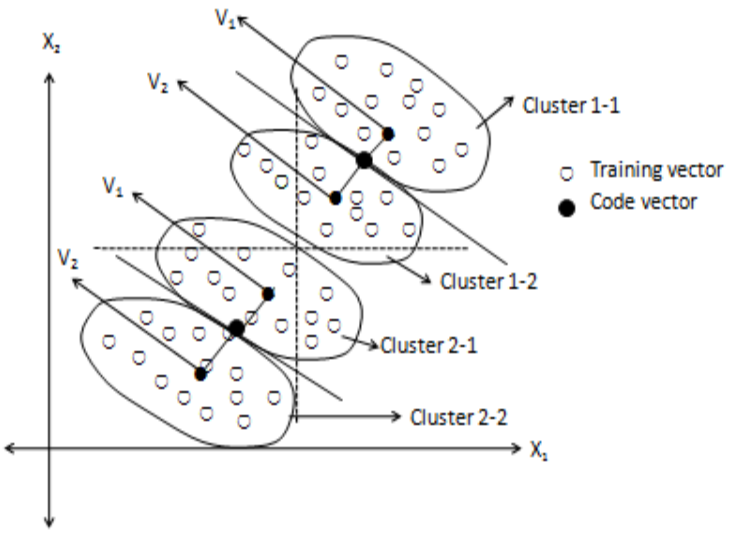

Figure 1(b) LBG for two dimensional case after four clusters formation

\section{PROPOSED ALGORITHM}

The proposed method can be divided into 3 sections. Section 3.1 explains generation of feature vector for training image. Same method is used to generate the feature vector of each testing image. Section 3.2 gives the procedure to generate 4,8 and 16 representative training feature vectors for each class using LBG vector quantization. Finally section 3.3 gives the method of classification of test image.

\subsection{Generation of Feature vector for each Training Image}

Image is resized to 256x256. Apply image transform ( such as DCT, DST, DFT, DHT, DWT and DKT) to the columns of $\mathrm{R}, \mathrm{G}$ and $\mathrm{B}$ plane of an image. Calculate the average of each row of a plane. This will generate the row mean vector for each plane. These row mean vectors are organized one below the other as shown in figure 2 to generate the feature vector. The size of each row mean vector is $256 \times 1$. The size of feature vector depends on the number of elements taken from the row mean vectors. If all elements of row mean vectors are taken, then the size of feature vector is $768 \times 1$. If only first 25 elements of each row mean vector is considered, then the feature vector of size $75 \mathrm{x} 1$ will be generated. Thus different sizes of feature vectors such as $150 \mathrm{x} 1,300 \mathrm{x} 1,450 \mathrm{x} 1,600 \mathrm{x} 1$ are also tried and tested their impact on the accuracy.

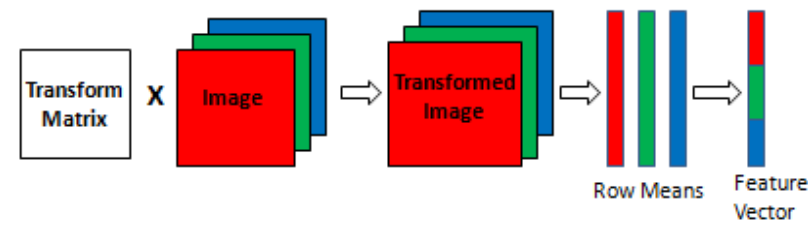

Figure 2. Generation of feature vector

\subsection{Generation of Representative Training Feature Vectors for Each Class}

Consider there are ' $\mathrm{N}$ ' training images in each class. Applying the procedure in section 3.1 will generate ' $\mathrm{N}$ ' training feature vectors for every class. Then, a well known LBG vector quantization method is applied to each class separately to generate codebooks of size 4,8 and 16 . The code vectors are used as representative for training feature vectors. Thus 4,8 and 16 feature vectors are generated for each class and used for training purpose. This procedure is shown in figure 3 . 


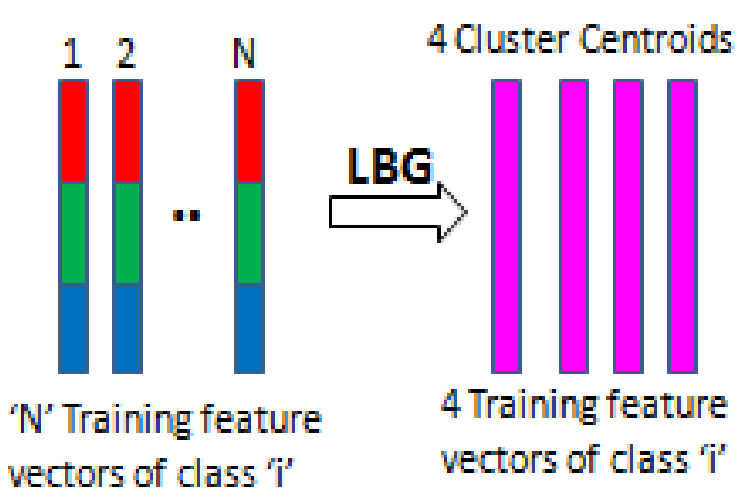

Figure 3. Code vector generation

\subsection{Classification of Test image}

For a test image, feature vector is generated using the procedure in section 3.1. Nearest neighbor algorithm is used for classification. The training feature vector closest to the test feature vector is found out and the test image is assigned to the corresponding class of that training feature vector as shown in figure 4. To find the closeness of feature vectors, different similarity criteria such as Euclidean distance, Manhattan distance, Cosine correlation and Bray-Curtis similarity are used.

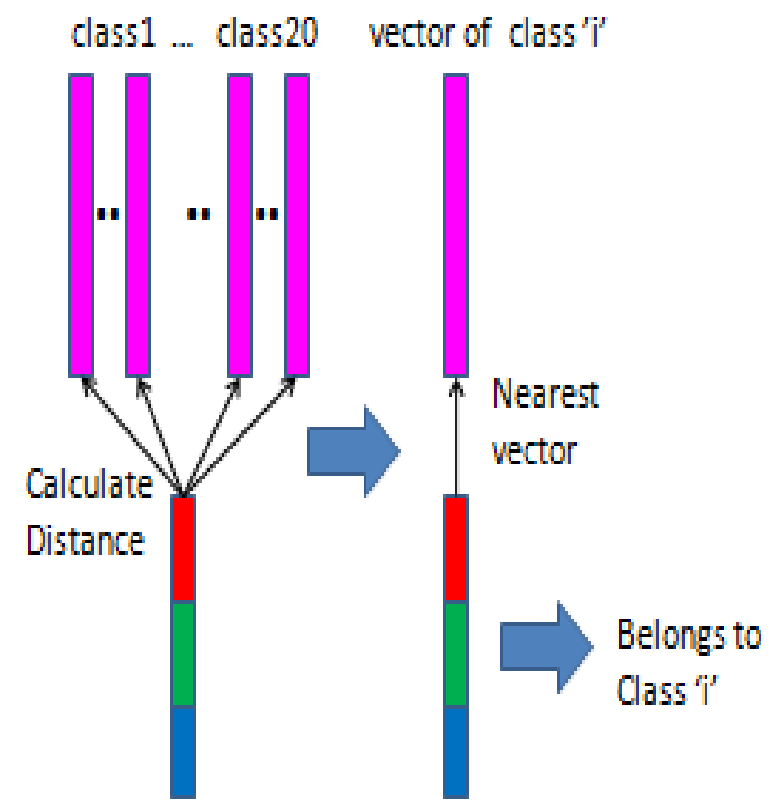

Fig.4. Classification of test image

\section{RESULTS AND DISCUSSION}

The implementation of the proposed method is done in MATLAB 7.0 using a computer with Intel Core i5, CPU $(2.50 \mathrm{GHz}$ and $6 \mathrm{~GB}$ RAM). The proposed techniques are tested on the Augmented Wang database (J.Z.Wang et al. 2001)[23]. Six classes (Dinosaur, Horse, Rose, Elephant, Mountains and Bus) are directly taken from Wang database. Remaining 14 classes are downloaded from internet. Each class contains 100 images. Total images in database are 2000. Figure 5 shows the sample images of Training database. Figure 6 shows the sample images of testing database.

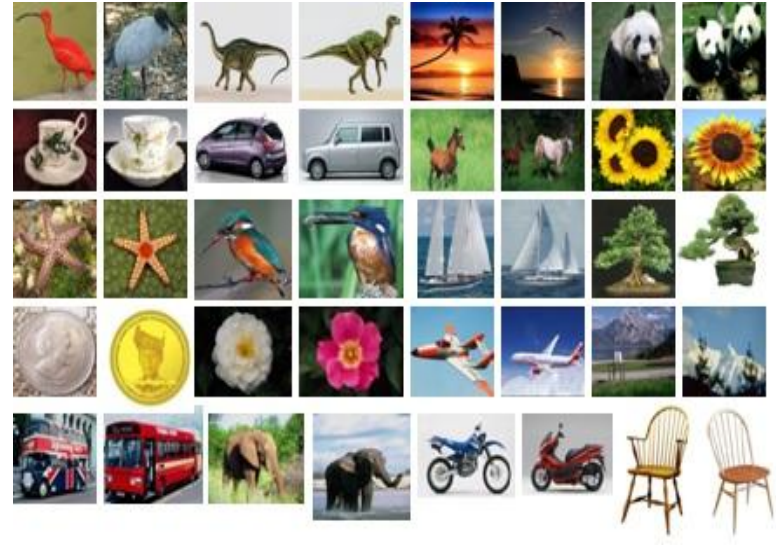

Figure 5. Sample Images of Training Database

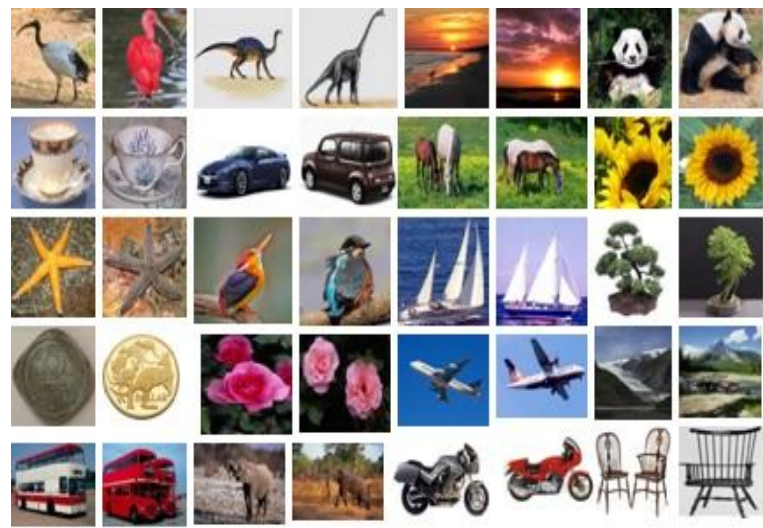

Figure 6. Sample Images of Testing Database

Initially 35 images per class are used for training. So total 700 images are used for training. Remaining 1300 images are used for testing. Table 1 shows the percentage accuracy of correctly classified images out of 1300 images for different transforms, different similarity measures. 'Average' indicates only one feature vector per class used for training purpose which is nothing but average of all training feature vectors in that class. 'All' indicates all 35 feature vectors per class are used for training purpose. LBG-4, LBG-8 and LBG-16 indicates the proposed vector quantization method with 4,8 and 16 code vectors used for training purpose. The algorithm is implemented for all feature vector sizes. But due to space constraint, we have given the results only for the feature vector of size $300 \times 1$. This size gives the highest overall accuracy. Later 45 images per class are used for training. Results are marginally improved. Overall highest accuracy achieved is $63.91 \%$ with transform-DST, feature vector size300x1 and Similarity Criteria -Bray-Curtis. Then 55 images per class are used for training purpose and remaining 45 images per class are used for testing purpose. So 1100 images are used for training and 900 images are used for testing. The performance in terms of accuracy is shown in table 2. Figure 7 indicates the accuracy results in each individual class for DST, Bray-Curtis similarity, feature vector size $300 \times 1$ and 55 training images per class. In 11 classes, LBG has shown improved performance over 'Average' and 'All' case. Figure 8 gives the accuracy results in each individual class for DCT when 55 images per class are used for training purpose. Table 3 shows the highest accuracy achieved for each transform for different number of training images and its corresponding similarity measure, feature vector size and classification method. 
Table 1. \%Accuracy of correctly classified images

Feature vector size $=100 \mathrm{G}+100 \mathrm{R}+100 \mathrm{~B}(300 \times 1)$

Training Images : 700

\begin{tabular}{|c|c|c|c|c|c|c|}
\hline \multirow{3}{*}{ Transform } & \multirow{3}{*}{$\begin{array}{c}\text { Similarity } \\
\text { Criteria }\end{array}$} & \multicolumn{5}{|c|}{ Number of training vectors/class } \\
\hline & & 1 & 4 & 8 & 16 & 35 \\
\hline & & (Average) & (LBG-4) & (LBG-8) & (LBG-16) & (All) \\
\hline \multirow{4}{*}{ DCT } & Euclidean & 47.23 & 52.85 & 55.31 & 55.54 & 57.69 \\
\hline & Manhattan & 52.54 & 55.46 & 58.92 & 60.46 & 57.23 \\
\hline & $\begin{array}{c}\text { Cosine } \\
\text { Correlation }\end{array}$ & 51.62 & 53.15 & 55.15 & 54.46 & 56.46 \\
\hline & Bray-Curtis & 52.15 & 56 & 59.85 & 60.62 & 57.46 \\
\hline \multirow{4}{*}{ DST } & Euclidean & 51 & 58.77 & 59.31 & 59.15 & 59.54 \\
\hline & Manhattan & 52.85 & 61.31 & 62.15 & 62.85 & 60.23 \\
\hline & $\begin{array}{c}\text { Cosine } \\
\text { Correlation }\end{array}$ & 55.15 & 59.62 & 62.08 & 61.38 & 61.38 \\
\hline & Bray-Curtis & 52.62 & 62.38 & 63.15 & 63.62 & 60.38 \\
\hline \multirow{4}{*}{ DFT } & Euclidean & 43.77 & 50.92 & 53.85 & 53.23 & 53.23 \\
\hline & Manhattan & 49.31 & 52.23 & 55.38 & 56.08 & 55.23 \\
\hline & $\begin{array}{c}\text { Cosine } \\
\text { Correlation }\end{array}$ & 48.69 & 51.46 & 53.77 & 52.92 & 51.54 \\
\hline & Bray-Curtis & 49.23 & 51.85 & 56.23 & 56.62 & 55 \\
\hline \multirow{4}{*}{ DHT } & Euclidean & 43.46 & 51.31 & 51.31 & 52.85 & 50.54 \\
\hline & Manhattan & 48.92 & 54 & 55.92 & 55.54 & 53.38 \\
\hline & $\begin{array}{c}\text { Cosine } \\
\text { Correlation }\end{array}$ & 47.54 & 49.38 & 50.92 & 50.08 & 49.46 \\
\hline & Bray-Curtis & 49 & 53.62 & 55.69 & 56.92 & 53.15 \\
\hline \multirow{4}{*}{ DWT } & Euclidean & 45.6 & 50.31 & 53.54 & 54.08 & 55.6 \\
\hline & Manhattan & 51.7 & 53 & 58 & 57.85 & 55.85 \\
\hline & $\begin{array}{c}\text { Cosine } \\
\text { Correlation }\end{array}$ & 51.31 & 51.46 & 53.77 & 53.62 & 55.38 \\
\hline & Bray-Curtis & 50.69 & 55.08 & 59.08 & 59.23 & 57 \\
\hline \multirow{4}{*}{ DKT } & Euclidean & 41.31 & 45.54 & 49.08 & 47.69 & 50.15 \\
\hline & Manhattan & 38.92 & 44.92 & 49.77 & 50.46 & 49.46 \\
\hline & $\begin{array}{c}\text { Cosine } \\
\text { Correlation }\end{array}$ & 45.69 & 45.15 & 46.92 & 45.54 & 50.31 \\
\hline & Bray-Curtis & 39.23 & 45 & 49.69 & 49.62 & 49.15 \\
\hline
\end{tabular}

Note : Yellow color indicates maximum accuracy achieved for each similarity criteria in each transform. Red color indicates overall maximum accuracy achieved for every transform.

Observations : LBG with code book size of 16 gives highest accuracy in all transforms. In Manhattan and Bray-Curtis similarity, this vector quantization method gives quite high improvement in accuracy. Highest accuracy achieved is
$63.62 \%$ with DST transform, Bray-Curtis similarity and with LBG (code book size 16). In DKT there is marginal improvement with vector quantization method when Manhattan distance is used. 
Table 2. \%Accuracy of correctly classified images

Feature vector size $=100 \mathrm{G}+100 \mathrm{R}+100 \mathrm{~B}(300 \times 1)$

Training Images : 1100

\begin{tabular}{|c|c|c|c|c|c|c|}
\hline \multirow{3}{*}{ Transform } & \multirow{3}{*}{ Similarity Criteria } & \multicolumn{5}{|c|}{ Number of training vectors/class } \\
\hline & & 1 & 4 & 8 & 16 & 55 \\
\hline & & (Average) & (LBG 4) & (LBG 8) & (LBG 16) & (All) \\
\hline \multirow{4}{*}{ DCT } & Euclidean & 47.78 & 54.44 & 58.67 & 59.56 & 60.44 \\
\hline & Manhattan & 54.44 & 59.67 & 61.7 & 65.9 & 61.89 \\
\hline & Cosine Correlation & 50.56 & 53.56 & 58.1 & 60.3 & 58.33 \\
\hline & Bray-Curtis & 54.44 & 60.11 & 60.6 & 65.6 & 62 \\
\hline \multirow{4}{*}{ DST } & Euclidean & 48.89 & 58.56 & 62.22 & 63.89 & 63 \\
\hline & Manhattan & 52.56 & 62.67 & 64.33 & 63.67 & 60.33 \\
\hline & Cosine Correlation & 54.56 & 60.44 & 63 & 63.89 & 61.78 \\
\hline & Bray-Curtis & 51.56 & 63.44 & 65.44 & 64.78 & 61.67 \\
\hline \multirow{4}{*}{ DFT } & Euclidean & 43.78 & 53.33 & 55.89 & 54.67 & 55.33 \\
\hline & Manhattan & 49.78 & 54.22 & 58.7 & 60 & 56.78 \\
\hline & Cosine Correlation & 50.56 & 52.33 & 54.4 & 57.4 & 53.89 \\
\hline & Bray-Curtis & 48.89 & 55 & 59.2 & 60.1 & 56.67 \\
\hline \multirow{4}{*}{ DHT } & Euclidean & 48.11 & 52.89 & 54.56 & 54.44 & 53.22 \\
\hline & Manhattan & 49.67 & 54 & 58.89 & 58 & 55.78 \\
\hline & Cosine Correlation & 51.89 & 50.22 & 53 & 52.56 & 52.56 \\
\hline & Bray-Curtis & 49.44 & 54 & 58.22 & 56.56 & 56.22 \\
\hline \multirow{4}{*}{ DWT } & Euclidean & 47 & 52.56 & 56.78 & 58.56 & 60.67 \\
\hline & Manhattan & 52.67 & 57.2 & 61.4 & 62.67 & 58.22 \\
\hline & Cosine Correlation & 51 & 53.4 & 57.6 & 57 & 57.11 \\
\hline & Bray-Curtis & 52.56 & 58.3 & 61.1 & 63.11 & 60.67 \\
\hline \multirow{4}{*}{ DKT } & Euclidean & 41.89 & 45.78 & 49.67 & 49 & 51.89 \\
\hline & Manhattan & 38.67 & 46.33 & 47.33 & 51.33 & 52.89 \\
\hline & Cosine Correlation & 46.22 & 45.78 & 48.22 & 47.67 & 52 \\
\hline & Bray-Curtis & 38.11 & 45.67 & 47.11 & 51.44 & 51.67 \\
\hline
\end{tabular}

Note : Yellow color indicates maximum accuracy achieved for each similarity criteria in each transform. Red color indicates overall maximum accuracy achieved for every transform

Observations : In all transforms except DKT transform, highest accuracy is achieved with LBG vector quantization. In Manhattan and Bray-Curtis similarity, this vector quantization method gives quite high improvement in accuracy in most cases. Highest accuracy achieved is $65.6 \%$ with DCT transform, Bray-Curtis similarity and with LBG (code book size 16) 


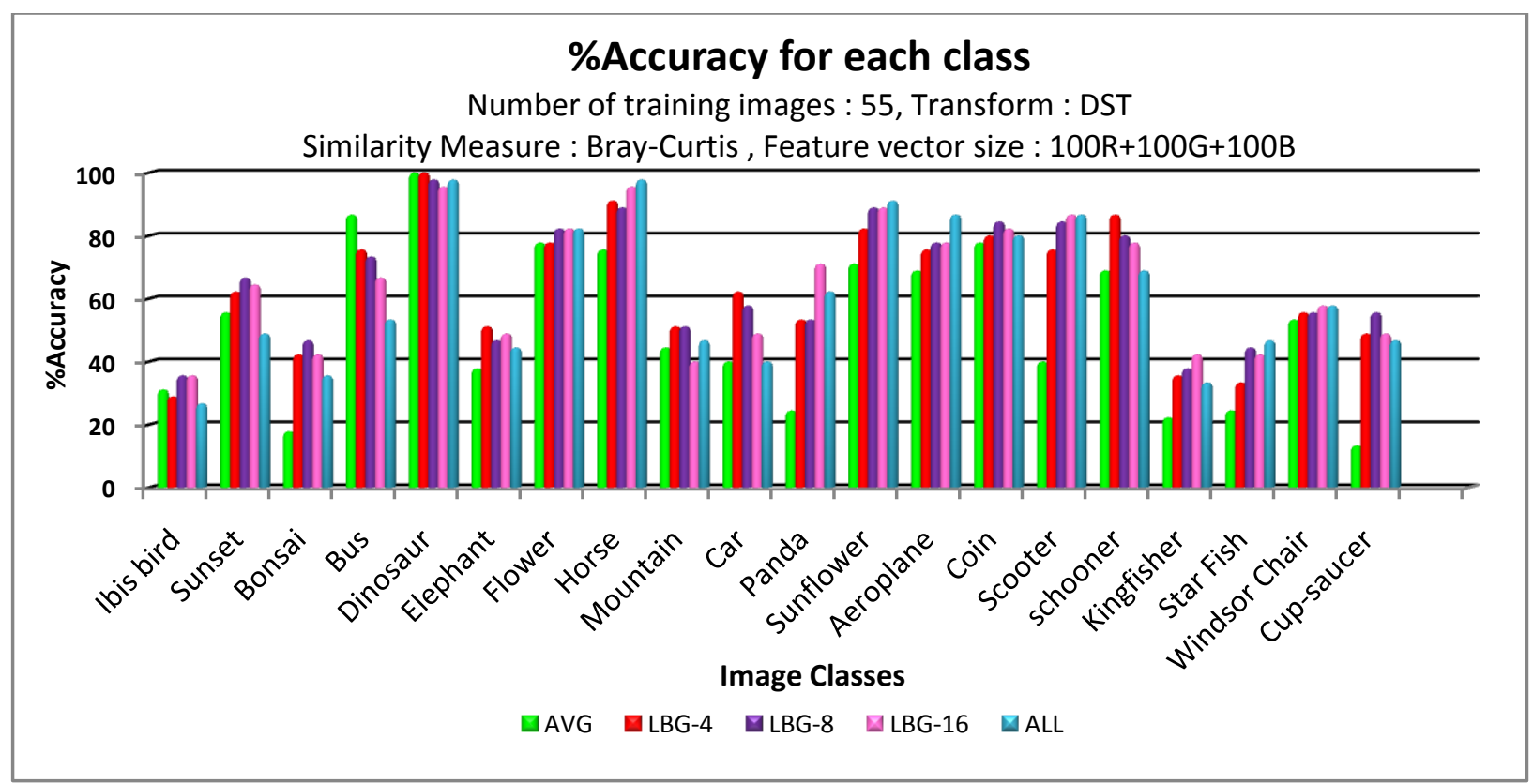

Figure 7. \% Accuracy for each individual class

Observations: In most of the individual classes such as Ibis bird, Sunset, Bonsai, Elephant, Mountain, Car, Panda, Coin, Schooner, Kingfisher and Cup-saucer, LBG gives higher accuracy than 'AVG' and 'ALL' case. In 11 classes, the maximum accuracy achieved is more than $60 \%$



Figure 8. \% Accuracy for each individual class

Observations: In most of the individual classes such as Ibis bird, Sunset, Bonsai, Dinosaur, Flower, Mountain, Car, Coin, Schooner, Kingfisher, Windsor Chair and Cup-saucer, LBG gives higher accuracy than 'AVG' and 'ALL' case. In 14 classes, the maximum accuracy achieved is more than $60 \%$. 
Table 3. Overall highest \%accuracy for each transform

\begin{tabular}{|c|c|c|c|c|}
\hline \multirow[t]{2}{*}{ Transform } & \multicolumn{4}{|c|}{ Number of training images/ number of testing images } \\
\hline & $\begin{array}{l}500 / 1500 \\
(25 \text { per class/ } \\
75 \text { per class })\end{array}$ & $\begin{array}{l}700 / 1300 \\
(35 \text { per class/ } \\
65 \text { per class })\end{array}$ & $\begin{array}{l}900 / 1100 \\
(45 \text { per class/ } \\
55 \text { per class })\end{array}$ & $\begin{array}{l}1100 / 900 \\
(55 \text { per class/ } \\
45 \text { per class })\end{array}$ \\
\hline DCT & $\begin{array}{l}\mathbf{5 9 . 3} \\
\text { Bray-Curtis } \\
300 X 1 \\
\text { LBG-8 }\end{array}$ & $\begin{array}{l}\mathbf{6 0 . 6 2} \\
\text { Bray-Curtis } \\
300 X 1 \\
\text { LBG-16 }\end{array}$ & $\begin{array}{l}62 \\
\text { Bray-Curtis } \\
450 X 1 \\
\text { LBG-16 }\end{array}$ & $\begin{array}{l}\mathbf{6 5 . 8 9} \\
\text { Bray-Curtis } \\
300 X 1 \\
\text { LBG-16 }\end{array}$ \\
\hline DST & $\begin{array}{l}61.8 \\
\text { Bray-Curtis } \\
450 X 1 \\
\text { LBG-8 }\end{array}$ & $\begin{array}{l}\mathbf{6 3 . 6 2} \\
\text { Bray-Curtis } \\
300 X 1 \\
\text { LBG-16 }\end{array}$ & $\begin{array}{l}\mathbf{6 3 . 9 1} \\
\text { Bray-Curtis } \\
300 X 1 \\
\text { LBG-16 }\end{array}$ & $\begin{array}{l}\mathbf{6 5 . 4 4} \\
\text { Bray-Curtis } \\
300 X 1 \\
\text { LBG-8 }\end{array}$ \\
\hline DFT & $\begin{array}{l}\mathbf{5 5 . 5} \\
\text { Bray-Curtis } \\
300 X 1 \\
\text { LBG-8 }\end{array}$ & $\begin{array}{l}\mathbf{5 6 . 6 2} \\
\text { Bray-Curtis } \\
300 X 1 \\
\text { LBG-16 }\end{array}$ & $\begin{array}{l}\mathbf{5 8 . 5 5} \\
\text { Bray-Curtis } \\
150 X 1 \\
\text { LBG-16 }\end{array}$ & $\begin{array}{l}\mathbf{6 0 . 6 7} \\
\text { Manhattan } \\
\text { Bray-Curtis } \\
150 X 1 \\
\text { LBG-16 }\end{array}$ \\
\hline DHT & $\begin{array}{l}\mathbf{5 6 . 4} \\
\text { Bray-Curtis } \\
300 X 1 \\
\text { LBG-8 }\end{array}$ & $\begin{array}{l}\mathbf{5 8 . 0 8} \\
\text { Bray-Curtis } \\
768 X 1 \\
\text { LBG-16 }\end{array}$ & $\begin{array}{l}\mathbf{5 9 . 8 2} \\
\text { Bray-Curtis } \\
768 X 1 \\
\text { LBG-16 }\end{array}$ & $\begin{array}{l}\mathbf{5 9 . 2 2} \\
\text { Bray-Curtis } \\
768 X 1 \\
\text { LBG-16 }\end{array}$ \\
\hline DWT & $\begin{array}{l}\mathbf{5 9 . 4} \\
\text { Manhattan } \\
\text { 450X1 } \\
\text { LBG-8 }\end{array}$ & $\begin{array}{l}\mathbf{5 9 . 6 2} \\
\text { Bray-Curtis } \\
768 X 1 \\
\text { LBG-16 }\end{array}$ & $\begin{array}{l}\mathbf{6 2 . 4 5} \\
\text { Manhattan } \\
300 X 1 \\
\text { LBG-16 }\end{array}$ & $\begin{array}{l}\mathbf{6 3 . 2 2} \\
\text { Manhattan } \\
450 X 1 \\
\text { LBG-16 }\end{array}$ \\
\hline DKT & $\begin{array}{l}\mathbf{5 7} \\
\text { Manhattan } \\
768 X 1 \\
\text { LBG-16 }\end{array}$ & $\begin{array}{l}\mathbf{5 8 . 6 9} \\
\text { Euclidean } \\
768 X 1 \\
\text { ALL }\end{array}$ & $\begin{array}{l}\mathbf{5 9 . 2 7} \\
\text { Manhattan } \\
768 X 1 \\
\text { ALL }\end{array}$ & $\begin{array}{l}\mathbf{6 1 . 7 8} \\
\text { Manhattan } \\
768 X 1 \\
\text { LBG-16 }\end{array}$ \\
\hline
\end{tabular}

Note : Yellow color indicates highest accuracy among all the transforms. Green color indicates highest performance in each column. Blue color indicates highest performance in each row

Observations: For DCT, DST, DFT, DHT and DWT, proposed technique has given the highest accuracy for all cases.

\section{CONCLUSIONS AND FUTURE SCOPE}

The paper presents an approach to generate compact and effective training set from the given training set of feature vectors. After doing a lot of experimentation, we have observed that classification with all training vectors gives far better results than single average training feature vector for each class. This fact has given an idea to make groups of training vectors of same class and select one representation for each group. This will keep the impact of all training vectors and also reduce the number of training vectors per class. For grouping, we have implemented LBG vector quantization with 4,8 and 16 code vectors for each class. Size of code book is limited to 4,8 and 16 because the minimum number of training images used is 25 . In the proposed technique LBG-4, LBG-8 and LBG-16, approximately 80 (4 x 20 classes), 160 ( 8 X 20 classes) and 320 (16 x 20 classes) training feature vectors are used for training respectively instead of all 500 (25 images /class), 700(35 images /class), 
900 (45 images/class) and 1100 (55 images/class) training feature vectors. Thus the technique significantly reduces the storage space and computation time. It also increases an accuracy. The proposed technique is applied on the augmented Wang database. The results of proposed method shows a high improvement in accuracy with Manhattan and Bray-Curtis similarity. With Euclidean and Cosine correlation similarity measures, classification with all training feature vectors gives better results. In DKT, for 25 training images per class, LBG-16 gives highest accuracy. But for other cases of DKT, classification with all training vector achieves highest accuracy. Bray-Curtis similarity outperforms in most of the cases. In DHT and DKT, feature vector size of $768 \times 1$ gives overall better results. In other transforms, most of the cases feature vector size of 300x 1 gives highest accuracy. As the number of training images increases, accuracy also increases. For 25,35 , and 45 training images per class, DST gives highest accuracy in comparison with all other transforms and it is $61.8 \%, 63.62 \%$ and $63.91 \%$ respectively. In classification with 55 training images per class, DCT has given highest accuracy of $65.89 \%$.

\section{REFERENCES}

[1] A.Vailaya, A.jain and H.Zhang. "On Image Classification: City Images vs. Landscapes." Pattern Recognition, Published by Elsevier Science Ltd., Vol. 31, No. 12, Dec 1998, pp.1921-1935

[2] N.Manshor, A. R. A. Rahiman, M. Rajeswari and D.Ramchandram. "Feature Fusion in Improving Object Class Recognition." Journal of Computer Science, Vol. 8, Issue 8, 2012, pp.1321-1328

[3] H.Nakayama, T.Harada and Y.Kuniyoshi. "Scene Classification using Generalized Local Correlation," in Proc. of IAPR Conference on Machine Vision Applications, May-2009, Yokohama Japan, pp.195-198

[4] D.Choudhary, A.K.Singh, S.Tiwari and V.P.Shukla. "Performance Analysis of Texture Image Classification using Wavelet Feature." International Journal of Image, Graphics and Signal processing, Vol.5, No.1, Jan 2013, pp. 58-63

[5] W.H.Cho, I.S.Na, J.Y.Choi and T.H.lee. "Automatic Classification for Various Images Collections Using Two Stages Clustering Method." Open Journal of applied sciences, Vol.3, No.1B, Mar 2013, pp. 47-52

[6] O. Boiman, E. Shechtman, and M. Irani, "In Defense of Nearest-Neighbor Based Image Classification, " IEEE Conference on Computer Vision and Pattern Recognition (CVPR), June 2008

[7] M.W.Ashour, F.Khalid and M.A.Obaydee. "Supervised ANN classification for engg machined textures based on enhanced features extraction and reduction scheme," in Proc. of the international conference on Artificial Intelligence in Computer Science and ICT (AICS 2013), Nov 2013, Malaysia, pp. 71-80

[8] Li Fei-Fei and Pietro Perona. "A bayesian Hierarchical Model for learning natural scene categories," in Proc of IEEE conference on Computer Vision and Pattern Recognition, CVPR, Vol. 2, Jun 2005, pp. 524-531

[9] S.D.Madan Raja and A.Shanmugam. "ANN and SVM based War Scene Classification using Wavelet features: a comparitive study." Journal of computational Information systems, Vol.7, No.5, 2011, pp. 1402-1411
[10] O.Brigham and R.E.Morrow. "The Fast Fourier Transform, " Spectrum, IEEE, Dec 1967, Vol.4, Issue 12 , pp.63-70

[11] N.Ahmed, T.Natrajan and K.R.Rao. "Discrete Cosine Transform. " IEEE Transactions, Computers,Jan 1974, pp.90-93

[12] A.K.Jain. "A Fast Karhunen-Loeve Transform for a Class of Random Processes. "IEEE Transaction on Communication, Vol. COM-24, Sep-1976, pp.1023-1029

[13] H.B.Kekre and J.K.Solanki. "Comparitive Performanceof Various Trignometric Unitary Transforms for Transform Image Coding. " International Journal of Electronics, Vol. 44, No.3, 1978, pp. 305-315

[14] Hartley, R.V.L. “A More Symmetrical Fourier Analysis applied to Transmission Problems," in Proc of IRE 30, Mar-1942, pp.144-150

[15] J.L.Walsh. "A Closed Set of Orthogonal Functions." American Journal of Mathematics, Vol.45, 1923, pp. 524

[16] H.B.Kekre and S.D.Thepade. " Image Retrieval using Non Involutional Orthogonal Kekre's Transform." International Journal of Multidisciplinary Research And Advances in Engineering, IJMRAE, Vol.1, No.I, Nov. 2009, pp.189-203.

[17] E.Deza and M.Deza, "Dictionary of Distances," Elsevier, 16-Nov-2006 - 391 pages

[18] John P., Van De Geer, "Some Aspects of Minkowski distance", Department of data theory,Leiden University. RR-95-03.

[19] S.Santini and R.Jain, "Similarity Measures," IEEE Transactions on Pattern Analysis and Machine Intelligence, Vol.21, No.9,pp.871-883, Sept1999

[20] H.B.Kekre, T.K.Sarode and J.K.Save. "Effect of Distance Measures on Transform based Image Classification." International Journal of Engineering Science and Technology (IJEST), Vol.4, No.8, Aug. 2012, pp.3729-3742

[21] Y.Linde, A.Buzo and R.Gray. “ An Algorithm for Vector Quantizer Design. " IEEE Transactions on Communications, Vol.28, No.1, Jan 1980, pp. 84-95

[22] R.M.Gray. “ Vector Quantization. ” IEEE ASSP Mag., Apr. 1984, pp. 4-29

[23] J.Z.Wang, J.Li and G.Wiederhold. "SIMPLIcity: Semantics-sensitive Integrated Matching for Picture Libraries." IEEE Transaction on Pattern Analysis and Machine Intelligence, Vol 23, no. 9, 2001,pp. 947-963

\section{AUTHORS' PROFILES}

Dr. H. B. Kekre has received B.E. (Hons.) in Telecomm. Engineering. from Jabalpur University in 1958, M.Tech (Industrial Electronics) from IIT Bombay in 1960, M.S.Engg. (Electrical Engg.) from University of Ottawa in 1965 and Ph.D. (System Identification) from IIT Bombay in $1970 \mathrm{He}$ has worked as Faculty of Electrical Engineering and then HOD Computer Science and Engg. at IIT Bombay. For 13 years he was working as a professor and head in the Department of Computer Engg. at Thadomal Shahani Engineering. College, Mumbai. Now he is Senior Professor at MPSTME, 
SVKM's NMIMS University. He has guided $17 \mathrm{Ph} . \mathrm{Ds}$, more than 100 M.E./M.Tech and several B.E./ B.Tech projects. His areas of interest are Digital Signal processing, Image Processing and Computer Networking. He has more than 500 papers in National /International Conferences and Journals to his credit. He was Senior Member of IEEE. Presently He is Fellow of IETE and Life Member of ISTE Recently twelve students working under his guidance have received best paper awards and ten research scholars have beenconferred $\mathrm{Ph}$. D. Degree by NMIMS University. Currently 7 research scholars are pursuing Ph.D. program under his guidance.

Tanuja K. Sarode has Received Bsc.(Mathematics) from Mumbai University in 1996, Bsc.Tech.(Computer Technology) from Mumbai University in 1999, M.E. (Computer Engineering) from Mumbai University in 2004, currently Pursuing Ph.D. from Mukesh Patel School of Technology, Management and Engineering, SVKM's NMIMS University, Vile-Parle (W), Mumbai, INDIA. She has more than 10 years of experience in teaching. Currently working as Associate Professor in
Dept. of Computer Engineering at Thadomal Shahani Engineering College, Mumbai. She is life member of IETE, ISTE, member of International Association of Engineers (IAENG) and International Association of Computer Science and Information Technology (IACSIT), Singapore. Her areas of interest are Image Processing, Signal Processing and Computer Graphics. She has more than 100 papers in National /International Conferences/journal to her credit.

Jagruti K. Save has received B.E. (Computer Engg.) from Mumbai University in 1996, M.E. (Computer Engineering) from Mumbai University in 2004, currently Pursuing Ph.D. from Mukesh Patel School of Technology, Management and Engineering, SVKM's NMIMS University, Vile-Parle (W), Mumbai, INDIA. She is currently working as Associate Professor in Dept. of Computer Engineering at Fr. Conceicao Rodrigues College of Engg., Bandra, Mumbai. Her areas of interest are Image Processing, Neural Networks, Fuzzy systems, Data base management and Computer Vision. She has 9 papers in National /International Conferences/journal to her credit. 